\title{
TEMA -2018: Cefalea por abuso de medicamentos
}
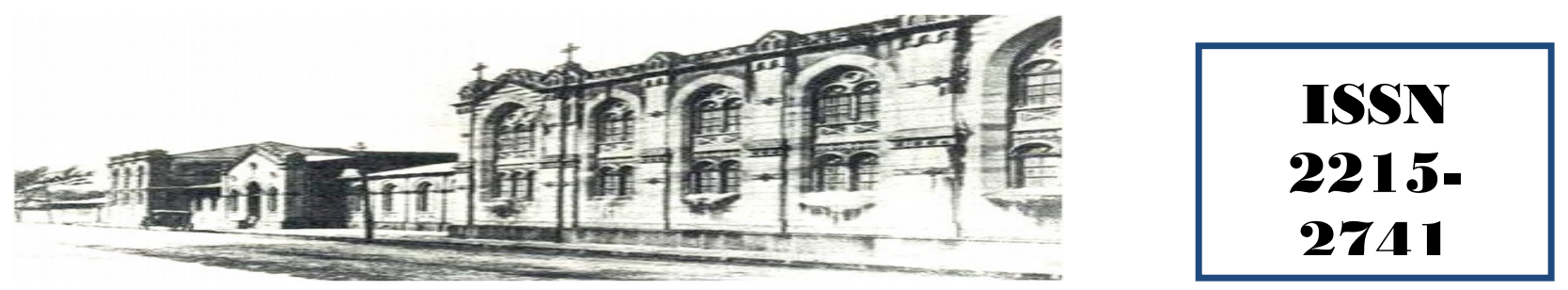

Haspital San quan de Dias. San José, Casta Rica. Fundada en 1845

$\begin{array}{ll}\text { Recibido: } & \text { 28/01/2018 } \\ \text { Aceptado: } & 15 / 03 / 2018\end{array}$

Luis Eduardo Gómez Víquez ${ }^{1}$

José Lee Chang Segura ${ }^{2}$

Huberth Fernández Morales ${ }^{3}$

${ }^{1}$ Médico General- Universidad de Costa Rica. Profesor de neuroanatomía: Universidad de Costa Rica. luis.gomezviquez@ucr.ac.cr

${ }^{2}$ Médico residente en neurología: Universidad de Costa Rica - Hospital Calderón Guardia. Profesor de neuroanatomía: Universidad de Costa Rica.

3Medico especialista en neurología: Hospital Calderón Guardia. Profesor de grado y postgrado, Escuela de Medicina, Universidad de Costa Rica.

\section{RESUMEN}

La cefalea por abuso de medicamentos corresponde a un fenómeno de cronificación y agravamiento de los síntomas en pacientes con cefaleas primarias, principalmente, migrañosa o tensional, quienes consumen con alta frecuencia, medicación para el alivio de las crisis dolorosas. Esta entidad alcanza una prevalencia del 1 al $2 \%$ en la población general. A continuación, se expone una revisión clínica de la etiología, fisiopatología, epidemiología, diagnóstico y manejo de este cuadro, altamente frecuente en la consulta médica general y especializada.

\section{PALABRAS CLAVE}

Cefalea. Abuso de medicamentos. Migraña tensional. Cefalea Secundaria. AINES. Tratamiento farmacológico.

\section{ABSTRACT}

Medication-overuse headache corresponds to a phenomenon of chronification and aggravation of symptoms in patients with primary headaches, mainly migraine or tension headache, who consume, frequently, relief drugs. This disorder reaches a prevalence around 1 to $2 \%$ in general population. The following is a clinical review of the etiology, pathophysiology, epidemiology, diagnosis and management of this condition, which is highly frequent in general and specialized medical consultations.

\section{KEY WORDS}

Headache. Medication-overuse. Tensional migraine. Secondary headache. NSAID. Pharmacological treatment. 
Tabla 1. Criterios diagnósticos de CAM ${ }^{(2)}$.

\section{INTRODUCCIÓN}

En pacientes con cefalea de larga data que recurren al uso de medicación sintomática con frecuencia, se puede desarrollar un cuadro de empeoramiento en la frecuencia o intensidad de los ataques, lo que se conoce actualmente como cefalea por abuso de medicamentos (anteriormente cefalea de rebote o cefalea medicamentosa). Esto genera un "círculo vicioso", donde se aumenta el consumo del tratamiento sintomático, a la vez que este se vuelve la causa de los síntomas. Dicho cuadro es más común en pacientes que padecen cefalea tensional o migraña.

La cefalea por abuso de medicamentos (CAM), es un problema común y que va en aumento, con una prevalencia aproximada del $1-2 \%$ en la población general ${ }^{(1)}$. Es un tipo de cefalea secundaria. La última versión de la clasificación internacional de las cefaleas (International classification of headache disorders. ICHD) la describe como una entidad donde se combina una cefalea de patrón crónico (más de 15 días por mes, por al menos 3 meses), con el abuso de medicación sintomática y que usualmente resuelve al descontinuar el fármaco. Este abuso está definido como el consumo de triptanos, opioides o analgésicos combinados 15 días o más al mes, o analgésicos simples (AINES/paracetamol) por 10 o más días al mes (2).

\section{DISCUSIÓN}

\section{Diagnóstico de CAM}

Los criterios diagnósticos para la CAM de la ICHD se resumen en la tabla 1 . El paciente debe cumplir los criterios A, B y C. Para fines de clasificación, se debe especificar el tipo de medicamento relacionado al diagnóstico (ej: "CAM por AINES"). En los casos que el paciente ya tenga un diagnóstico de cefalea primaria (ej: migraña, cefalea tensional) con el desarrollo de un nuevo tipo de cefalea o empeoramiento de la inicial, se determina un doble diagnóstico: la cefalea primaria y la CAM (2).
A. Cefalea por 15 o más días al mes con una cefalea preexistente.

B. Abuso por más de 3 meses de fármacos para el alivio o tratamiento sintomático de la cefalea*.

C. No es mejor explicado por otro diagnóstico de la ICHD-3.

*Definido por el consumo por $\geq 15$ días de triptanos. Opioides o ergotamínicos $o \geq 10$ días para AINES y paracetamol.

Fuente: adaptado de (2)

\section{Epidemiología y factores de riesgo}

La prevalencia de la CAM es de un $1-2 \%$, con una incidencia mayor en mujeres que en hombres relación aproximada de 3:1). Asimismo, es mayor la frecuencia de presentación en pacientes de edad mediana (40-50 años) que en aquellos más jóvenes ${ }^{(3)}$. En pacientes con cefalea crónica diaria, la prevalencia es mucho mayor y se ubica entre un $11-70 \%$. Un $80 \%$ de los casos de CAM corresponden a pacientes con cuadros migrañosos de fondo ${ }^{(4)}$.

Respecto al riesgo de desarrollo de CAM para distintos tipos de fármacos, revisiones sistemáticas han encontrado que el riesgo de desarrollo de CAM es menor en pacientes que utilizan triptanos y ergotamínicos respecto a la utilización de analgésicos simples ${ }^{(5)}$. A su vez, el uso de opioides tiene un riesgo mayor respecto al resto de grupos de medicamentos, con tasas de inducción superiores hasta en un $44 \%{ }^{(6)}$.

La incidencia de CAM es mucho mayor en pacientes que sufren de cefalea tensional y migraña. Es rara la presentación en cefaleas primarias de poca frecuencia crítica, como la cefalea en racimos, en las cuales el sujeto no tiene dificultad para descontinuar el uso de la medicación sintomática al resolverse el brote ${ }^{(7)}$.

\section{Fisiopatología}

La génesis de la CAM está relacionada con la activación del sistema trigémino-vascular y el sistema trigémino-cervical. La activación de estos está modulada por estructuras como fibras ascen- 
dentes del tallo cerebral (ej: sustancia gris periacueductal y núcleos del rafé). Asimismo, las influencias trigeminales generan actividad en centros de proyección como el tálamo o la corteza somatosensorial. Todas estas estructuras son susceptibles a fenómenos de neuroplasticidad y adaptación producto de la influencia de factores como la activación crónica o variaciones en sistemas de neurotransmisión ${ }^{(8,9)}$.

Los fármacos para alivio sintomático en cefalea inducen alteraciones en sistemas serotoninérgicos y endocanabinoides. Por ejemplo, en estudios con modelos animales, se ha demostrado que la exposición prolongada a fármacos como paracetamol o triptanes, induce una disminución en los niveles de serotonina en la corteza del lóbulo frontal (a expensas de un aumento en los transportadores de serotonina y disminución en los sitios de unión de receptores $5-\mathrm{HT}_{2 \mathrm{~A}}$ ). Estas alteraciones generan a su vez, un aumento en el fenómeno de propagación de la depresión cortical, importante en modelos experimentales de migraña ${ }^{(7,10)}$. De igual modo se ha demostrado la reducción en niveles de anandamida y 2-acilglicerol (cannabinoides endógenos) en pacientes con migraña crónica y $\mathrm{CAM}^{(11)}$.

Mediante estudios de neuroimágenes se ha determinado que en pacientes con CAM hay un aumento en el volumen de sustancia gris a nivel del mesencéfalo, tálamo y cuerpo estriado. Estos cambios podrían deberse a alteraciones en la modulación del dolor a nivel central. De acuerdo con los hallazgos en estos estudios, las alteraciones son reversibles y se normalizarían al descontinuar el fármaco desencadenante ${ }^{(10,12)}$.

En estudios de actividad metabólica por resonancia magnética funcional, se ha visto hipoactividad en zonas relacionadas al procesamiento del dolor (corteza parietal, zonas somatosensoriales, giro supramarginal) asociadas a consumo de medicación sintomática. Estos cambios serían reversibles al descontinuar los fármacos y se deben a la estimulación repetitiva del sistema trigeminal o bien a la acción directa de los medicamentos (13).

\section{Cuadro clínico}

Las características clínicas dependen de la cefalea de fondo del paciente. En sujetos con migra- ña, es posible que se manifiesten características típicas como la unilateralidad, foto/osmofobia, pulsatilidad y agravamiento con la actividad. De manera similar, en pacientes con cefalea tensional, el dolor opresivo, bilateral, en banda, puede ser la tónica ${ }^{(7.9)}$. No debe obviarse que la medicación puede modificar o enmascarar algunos de los síntomas de la cefalea primaria, por lo que no es de extrañar que en algunos o todos los días que se presenta cefalea, se reporten características variables respecto al cuadro episódico inicial. Otro fenómeno descrito en la literatura es el aumento de la intensidad del dolor posterior al consumo de analgésicos o la falta respuesta a los mismos ${ }^{(7)}$.

\section{Tratamiento}

Existen diversas propuestas terapéuticas con grados variables de evidencia. Estas combinan dos grandes líneas de acción: la retirada de la medicación sintomática y la instauración de terapia profiláctica para prevenir los ataques de la cefalea primaria (migraña, en la mayoría de casos). De manera paralela al retiro del medicamento, se puede o no requerir el manejo de la cefalea y los síntomas asociados al mismo.

\section{Suspensión de medicamentos.}

Esta medida es el pilar del tratamiento en la CAM, pues ha demostrado la reducción significativa de los síntomas con la utilización de estrategias que varían desde la educación al paciente, hasta programas de desintoxicación con manejo farmacológico, ya sean ambulatorios o intrahospitalarios $^{(14)}$.

\section{a) Educación al paciente.}

En un estudio prospectivo, aleatorizado, con 120 pacientes quienes tomaban medicación a bajas dosis, se comparó la efectividad de brindar educación al paciente sobre la reducción del consumo de medicamentos, contra un programa de desintoxicación estructurado tanto ambulatorio (manejo con esteroides y medicación profiláctica) como intrahospitalario (esteroides, terapia profiláctica, antieméticos y fluidos). Al comparar las tres modalidades se obtuvieron resultados similares en todos, con una tasa de éxito en la desintoxicación de $70 \%$ a 2 meses $(\mathrm{p}<0.01)^{(15)}$. 
Un estudio prospectivo desarrollado en Noruega, con 109 sujetos a quienes se brindó información escrita sobre el rol de los fármacos en la generación la cefalea, obtuvo resultados positivos respecto a reducción en el uso de medicación. Se pasó en promedio de 22 a 6 días por mes de uso de fármacos y en $76 \%$ de los casos se obtuvo retirada completa de los medicamentos ${ }^{(16)}$.

Diversos autores han recomendado que el abordaje, únicamente con educación, puede intentarse como una primera opción en pacientes quienes toman fármacos a bajas dosis y sin otros factores de complejidad, como patologías psiquiátricas, enfermedades crónicas o el uso de opioides, ansiolíticos u otros ${ }^{(7)}$.

b) Estrategias de retiro abrupto y escalonado de fármacos.

A la fecha no se cuenta con estudios de alto poder estadístico que comparen directamente ambas estrategias. Algunos especialistas, basados en su experiencia clínica, recomiendan la suspensión abrupta del fármaco, pues se cree de esta forma se alcanza de manera más rápida una mejoría en los síntomas ${ }^{(17)}$. Medicamentos como AINES, paracetamol, triptanos y ergqotamínicos, se pueden suspender de forma repentina sin generar síntomas severos de abstinencia ${ }^{(14,17,18)}$.

En el caso de opioides se recomienda la disminución escalonada. Si se utilizan bajas dosis, se puede reducir la dosis a la mitad inicialmente y luego suspender en 1-2 semanas. Otra estrategia consiste en la suspensión del opioide y uso de sustitutos de acción prolongada como metadona oral (10-20 mg 2 veces/día), con reducción a la mitad en 3-5 días y suspensión en un periodo similar ${ }^{(9,19)}$. Asimismo, se puede recurrir al uso de parches de clonidina o la administración oral de la misma (0,1-0,2 mg 3 veces al día) para tratar los síntomas adrenérgicos por abstinencia de opioides $^{(20)}$.

De manera similar, se debe hacer un descenso paulatino en el caso de las benzodiacepinas y barbitúricos. Para estos últimos, se recomienda la suspensión y cambio por dosis equivalentes de fenobarbital con descenso de $30 \mathrm{mg}$ cada 2-3 días ${ }^{(20)}$. c) Manejo de los síntomas por abstinencia
(terapia puente).

Los principales síntomas de abstinencia de la medicación sintomática incluyen el empeoramiento de la cefalea, náuseas, vómito, hipotensión, taquicardia, ansiedad y trastornos del sueño. Estos síntomas usualmente duran de 2 a 10 días, y suelen ser menores para el uso de triptanes respecto a los AINES o ergotamínicos ${ }^{(17)}$. La "terapia puente" consiste en una serie de medidas que buscan prevenir o mitigar estos síntomas.

Una de las terapias propuestas para el manejo de la cefalea por abstinencia de fármacos es el uso de esteroides. En dos estudios que compararon el uso de esteroides vs. placebo, no se encontró diferencia significativa en la reducción de síntomas; dentro de las limitantes que tienen estos ensayos se encuentra la posibilidad de esquemas a dosis y duraciones bajas ${ }^{(17,20)}$. Por otra parte, existen estudios pequeños que sí han encontrado beneficios en el uso de esteroides, principalmente en la reducción de la cantidad de horas con cefalea para pacientes con migraña ${ }^{(21)}$. De momento, la evidencia es escaza y ambigua respecto a este tipo de fármacos. Algunos esquemas recomiendan el uso de ciclos cortos de esteroides, por ejemplo, prednisona oral $60-100 \mathrm{mg}$ /día por 5 días ${ }^{(20)}$.

Otro medicamento que se ha utilizado para mitigar los síntomas por abstinencia es el naproxeno (a dosis de $550 \mathrm{mg}$ 1-2 veces al día) Solo se ha probado en estudios pequeños no controlados y por periodos de 1-2 semanas ${ }^{(18)}$. Para el manejo de los vómitos severos se puede recurrir al uso de antieméticos (como metoclopramida o domperidona) y fluidos intravenosos. En pacientes con cefalea de características migrañosas y sin abuso de ergotamínicos, se puede recurrir a la dihidroergotamina IV (1-2 mg c/8 horas) ${ }^{(17)}$. En caso de requerirse analgesia de rescate, se recomienda utilizar un medicamento diferente al que haya usado el paciente con anterioridad.

Como ya se mencionó, en el caso de opioides, benzodiacepinas y barbitúricos, se debe seguir un descenso escalonado y valorar la sustitución con drogas de vida media larga para prevenir síndromes de abstinencia severos

d) Terapia ambulatoria vs. hospitalaria. 
Comparaciones directas entre los dos esquemas, han mostrado reducciones significativas en el número de días por mes con cefalea a 1 año para ambos. No se ha encontrado superioridad significativa de uno sobre otro ${ }^{(22)}$.

Un estudio prospectivo, en 137 casos de CAM complicada (definida como aquella que presenta además comorbilidades psiquiátricas, enfermedades crónicas, fallo previo a la desintoxicación, uso de opiodes u otras sustancias psicoactivas), se obtuvo una reducción significativamente mayor en el grupo de desintoxicación intrahospitalaria $(89 \%$ vs $60 \%$ en los otros dos grupos, $p$ $<0.01)^{(23)}$.

Basados en estos estudios, y en recomendaciones de expertos, algunos autores señalan la conveniencia de la terapia extrahospitalaria en la mayoría de los pacientes, principalmente en pacientes con adecuada educación y motivación y con uso moderado de drogas como AINES, paracetamol y triptanos ${ }^{(7)}$. Los casos donde se recomienda indicar una terapia intrahospitalaria son ${ }^{(19)}$ :

- Uso de opioides, benzodiacepinas o barbitúricos.

- Vómitos o deshidratación severos.

- Fallo previo a terapia ambulatoria.

- Comorbilidades psiquiátricas.

- Comorbilidades orgánicas (ej: diabetes).

\section{Medicación profiláctica.}

Se recomienda la medicación profiláctica ya sea durante o después de completada la desintoxicación, principalmente en pacientes con migraña (que dan cuenta de la mayoría de casos de CAM). No existe evidencia científica de peso que compare la efectividad del inicio durante o posterior a completar el retiro del fármaco. Actualmente, hay estudios aleatorizados controlados, en pacientes quienes cumplen los criterios de clasificación de CAM, solo para el uso de topiramato (50 mg BID) e inyecciones de toxina botulínica tipo A, en pacientes con cefalea migrañosa de fondo ${ }^{(7)}$. Varios estudios observacionales de poco poder han demostrado beneficios en el uso de terapias como valproato, pregabalina, estimulación o bloqueo occipital, cannabinoides, entre otros. Debido a la ausencia de estudios de alto poder estadístico, actualmente no se pueden ha- cer recomendaciones respecto a estas terapias $(7,24)$.

Teniendo como objetivo la reducción en el consumo de medicación abortiva de las crisis, algunos autores señalan que se puede recurrir al uso de medicamentos profilácticos para migraña episódica, aun cuando estos no hayan sido probados propiamente en casos de traslape con CAM ${ }^{(20)}$. Tales medicamentos incluyen el uso de betabloqueadores inespecíficos como el propranolol, anticonvulsivantes como el valproato y topiramato, antidepresivos tricíclicos como amitriptilina e imipramina, los cuales cuentan con evidencia suficiente para su recomendación en migraña episódica ${ }^{(25)}$.

De forma similar, en el caso particular del traslape entre migraña tensional y CAM, se recomienda el uso de antidepresivos tricíclicos como amitriptilina o imipramina $(10-150 \mathrm{mg} / \mathrm{d} \text { ía })^{(17,26)}$. En la figura 1 se propone un resumen del manejo de la CAM.

\section{CONCLUSIONES}

La cefalea por abuso de medicamentos es una entidad claramente descrita y de alta prevalencia en pacientes con cefaleas de tipo migraña y tensional. Todos los medicamentos para el tratamiento agudo de estas entidades poseen el potencial de generar el cuadro.

Fisiopatológicamente se han evidenciado alteraciones, estructurales y metabólicas, en estructuras centrales de procesamiento de estímulos nociceptivos y sistemas de neurotransmisores, ambos relacionados con el consumo de la medicación causal.

El tratamiento de la CAM se fundamenta en el retiro del medicamento causante. No existe evidencia suficiente para diferir entre las distintas estrategias, pero los expertos favorecen la retirada abrupta cuando no existen complicaciones como comorbilidades psiquiátricas, consumo de opioides, benzodiacepinas u otros. Asimismo, se recomienda la medicación y la instauración de un esquema profiláctico de control de la cefalea primaria para evitar las recaídas del cuadro. 


\section{BIBLIOGRAFÍA}

1. Kristoffersen E Lundqvist C. Medicationoveruse headache: epidemiology, diagnosis and treatment. Therapeutic Advances in Drug Safety. 2014; 5(2): 8-99.

2. IHS. III edición de la clasificación internacional de las cefaleas UK: SAGE; 2013.

3. Westergaard M Hansen E Glumer C Olesen J Jensen R. Definitions of medication-overuse headache in population-based studies and their implications on prevalence estimates: A systematic review. Cephalalgia. 2013; 34(6): 409-425.

4. Westergaard M Hansen E Glumer C Olesen J Jensen R. Prevalence of chronic headache with and without medication overuse: associations with socioeconomic position and physical and mental health status. Pain. 2014; 155: 20052013.

5. Thorlund K Sun-Edelstein C Kanters E,et al. Risk of medication overuse headache across classes of treatments for acute migraine. The journal of Headache and Pain. 2016; 17(107): 696-698.

6. Lipton R. Risk Factors for and Management of Medication-Overuse Headache. Continuum. 2015; 21(4): 1181-31.

7. Diener H Holle D Solbach K Gaul C. Medication-overuse headache: risk factors, pathophysiology and management. Nat Rev Neurol. 2016; 12: $575-583$.

8. Jay G Barkin R. Primary Headache Disorders- Part 2: Tension-type headache and medication overuse headache. Dis Mon. 2017; 17.

9. Ropper A Samuels M Klein J. Adams y Victor Principios de Neurología. In.: McGraw Hill; 2016: 168-197.

10. Srikiatkhachorn A Maneesri S Supornsilpchai W Storer J. Pathophysiology of Medication Overuse Headache-An Update. Headache. 2014; 205:204-10.
11. Rossi C Pini L Cupini M Sarchielli P. Endocannabinoids in platelets of chronic migraine patients and medication-overuse headache patients: relation with serotonin levels. Eur. J. Clin. Pharmacol. 2008; 64: 1-8

12. Todd J Schwedt Chong. Medication Overuse Headache: Pathophysiological Insights from Structural and Functional Brain MRI Research. Headache. 2017; 130 (37): 1173-1178.

13. Ferraro S Grazzi L Mandelli $\mathrm{M}$ et al. Pain processing in medication overuse headache: a functional magnetic resonance imaging (fMRI) study. Pain Med. 2012; 13(2): 255-262.

14. Longo D Kasper D Jameson J Fauci A Hauser S Loscalzo J. Harrison principios de medicina interna. 18th ed. Mexico: McGraw Hill; 2012.

15. Rossi P Di Lorenzo C Faroni J Cesarino F Nappi G. Advice alone vs. structured detoxification programmes for medication overuse headache: a prospective, randomized, open-label trial in transformed migraine patients with low medical needs. Cephalalgia. 2006; 26: 1097-105.

16. Grande $\mathrm{R}$ Aaseth $\mathrm{K}$ Benth $\mathrm{J}$ Lundqvist $\mathrm{C}$ Russell M. Reduction in medication-overuse headache after short information. The Akershus study of chronic headache. Eur J Neurol. 2011; 18(1): 129-137.

17. Abrams B. Medication Overuse Headaches. Med Clin North Am. 2013; 97(2): 337-352

18. Evers S Jensen R. Treatment of medication overuse headache - guideline of the EFNS headache panel. Eur J Neurol. 2011; 18: 111521.

19. Paemeleire K Crevits L Goadsby P Kaube H. Practical management of medication-overuse headache. Acta neurol. belg. 2006; 106: 43-51.

20. Garza I Schwedt T. Medication overuse headache: treatment and prognosis. Up to Date. [Online].; 2016 [cited 2017 Noviembre 27. Disponible en: https://www-uptodatecom.ezproxy.sibdi.ucr.ac.cr/contents/medicationoveruse-headache-treatment-and-prognosis? source $=$ search_result\&search $=$ medication $\% 20$ overuse $\% \overline{2}$ headache $\&$ selectedTitle $=2 \sim 74$. 
21. Pageler L Katsarava Z Diener H Limmroth V. Prednisone vs. placebo in withdrawal therapy following medication overuse headache. Cephalalgia. 2008; 28(2): 152-156.

22. Evers S Marziniak M. Clinical features, pathophysiology, and treatment of medicationoveruse headache. Lancet Neurol. 2010; 9: 391401.

23. Rossi P Faroni J Tassorelli C Nappi G. $A d$ vice alone versus structured detoxification programmes for complicated medication overuse headache (MOH): a prospective, randomized, open-label trial.. J Headache Pain. 2013; 14(10): 1-8.

24. Chinag $\mathrm{C}$ Schwedt $\mathrm{T}$ Wang $\mathrm{S}$ Dodick D. Treatment of medication-overuse headache: $A$ systematic review. Cephalalgia. 2016; 1(16): 371-86.

25. Silberstein S. Practice parameter: evidencebased guidelines for migraine headache (an evidence-based review): report of the Quality Standards Subcommittee of the American Academy of Neurology. Neurology. 2000; 55: 754-762.

26. Westover M, Choi E, Awad K, Bianchi M. Pocket neurology. 2nd ed. Massachusetts: Wolters Kluwer; 2016.

\section{CONFLICTO DE INTERÉS Y/O AGRADECIMIENTOS}

Los autores declaran que no existió ningún conflicto de interés en el presente reporte. 


\section{Figura 1. Manejo de la CAM.}

\section{Fuente: propia}

Cefalea por abuso de medicamentos

\section{Educación al paciente}

\begin{tabular}{|c|c|c|}
\hline $\begin{array}{l}\text { ¿Vómitos/deshidratación severos? } \\
\text { ¿Fallo previo a terapia ambulatoria? }\end{array}$ & \multirow[b]{3}{*}{ SÍ } & \multirow{2}{*}{$\begin{array}{l}\text { ¿Abuso de opiodes, benzodiacepinas } \\
\text { o barbitúricos? }\end{array}$} \\
\hline ¿Comorbilidades psiquiátricas.? & & \\
\hline ¿Comorbilidades orgánicas? & & \multirow{4}{*}{$\begin{array}{l}\text { Considerar hospitalización } \\
\text { 1. Opioides: Dosis bajas: } \downarrow \\
\text { dosis a la } 1 / 2 \text { y luego } \mathrm{s} / \mathrm{s} \text { en } 1-2 \\
\text { sem. Dosis alta: sust. con } \\
\text { metadona } 10-20 \mathrm{mg} \mathrm{BID} \downarrow \text { a la } \\
1 / 2 \text { c/3-5 dias. Clonidina: } \\
\text { parches } 24 \mathrm{~h} 00,1-0,2 \mathrm{mg} \text { TID } \\
\text { V0 } \\
\text { 2. Barbitúricos: sustitución con } \\
\text { fenobarbital } \downarrow \downarrow 30 \mathrm{mg} \mathrm{c} / 2-3 \mathrm{~d}\end{array}$} \\
\hline $\begin{array}{l}\text { Manejo } \\
\text { ambulatorio }\end{array}$ & $\begin{array}{l}\text { Manejo } \\
\text { hospitalario }\end{array}$ & \\
\hline $\begin{array}{l}\text { - S/S abrupta de fármaco. } \\
\text { - Prednisona } 60-100 \mathrm{mg} / \text { dia } \times 5 \mathrm{~d} \\
\text { - Naproxeno } 550 \mathrm{mg} \text { BID PRN 1-2 sem } \\
\text { - Antiemético oral PRN } \\
\text { - Ansioliticos PRN }\end{array}$ & \multirow{2}{*}{$\begin{array}{l}\text { - S/S abrupta del fármaco. } \\
\text { - Fluidos IV. } \\
\text { - Antieméticos/ansioliticos IV/oral PRN. } \\
\text { - Prednisona 60-100 mg/dia x 5d } \\
\text { - Naproxeno } 550 \mathrm{mg} \text { BDD PRN 1-2 sem } \\
\text { - Dihidroergotamina IV 1-2 mg TID (pte. } \\
\text { sin uso previo). }\end{array}$} & \\
\hline & & \\
\hline \multicolumn{3}{|c|}{$\begin{array}{l}\text { Profilaxis: } \\
\text { Cefalea migrañosa: Topiramato } 50 \mathrm{mg} \text { BDD. Amitriptilina/Imipramina } 10-150 \mathrm{mg} / \text { dia. Propranolol } 40-240 \mathrm{mg} / \text { dia. Valproato } 500-2000 \mathrm{mg} / \text { dia. } \\
\text { Inyecciones toxina botulinica. }\end{array}$} \\
\hline
\end{tabular}

